\title{
Perfil psicológico de gênero, qualidade de vida e depressão: proposta de um modelo causal em mulheres idosas
}

\author{
Psychological profile of gender, quality of life and depression: \\ a proposal for a causal model in elderly women.
}

\author{
Marcela Cipriani Rufine ${ }^{[a]}$, Amanda Alves da Silva $a^{[b]}$, Nilton Soares Formiga ${ }^{[\mathrm{cl}}$, Gislane Ferreira de Melo ${ }^{[\mathrm{dd}]}$
}

\footnotetext{
[a] Doutora em Educação Física pela Universidade Católica de Brasília, DF - Brasil, e-mail:

mfcipriani@gmail.com
}

\section{Resumo}

Este estudo pretende verificar a associação entre perfil psicológico de gênero, nível de depressão e qualidade de vida em uma amostra de 143 idosas. Utilizando-se da análise de equações estruturais verificou-se inicialmente um modelo geral, o qual não se mostrou adequado devido à existência de saturações negativas entre os fatores e seus constructos. Optou-se então por um modelo parcimonioso, considerando individualmente as variáveis relacionadas ao perfil de gênero e gerando modelos mais específicos para cada variável da masculinidade e feminilidade como explicadores da qualidade de vida e da depressão. Observou-se, no que concerne ao modelo geral do perfil de gênero, que a norma masculina apresentou escores associativos com a qualidade de vida e a depressão, porém, essas duas últimas variáveis associaram-se negativamente. No que diz respeito ao modelo geral do perfil de gênero em relação à norma feminina, houve uma associação positiva com a qualidade de vida e, negativamente, com a depressão. Conclui-se que em todos os modelos propostos a qualidade de vida foi capaz de inibir a depressão, especialmente quando o construto da qualidade de vida contempla dimensões como capacidade funcional, aspectos físicos, aspectos emocionais e aspectos sociais, independentemente do perfil de gênero.

Palavras-Chave: Qualidade de Vida. Depressão. Idosas. Gênero.
Recebido: 09/11/2012

Received: 11/09/2012

Aprovado: 23/07/2013

Approved: 07/23/2013

\begin{abstract}
This study aims to investigate the association between psychological gender, level of depression and quality of life in a sample of 143 elderly women. Using structural equation analysis, there was initially a general model which was not suitable due to the existence of negative saturations between factors and their constructs. Then it was chosen to use a parsimonious model considering individually the variables related to gender profile and generating more specific models for each variable of masculinity and femininity as predictors of quality of life and depression. Referring to the general model of gender profile, it was found that the male norm scores showed associations with quality of life and depression, but these last two variables were associated negatively. Regarding the general model of the profile of gender in relation to the standard female, there was a positive association with quality of life and negatively association with depression. It
\end{abstract}


was reckoned that in all models proposed quality of life was able to inhibit depression, especially when the construct of quality of life includes dimensions such as functional, physical, emotional and social aspects of the profile, independently of gender.

Keywords: Quality of Life. Depression. Elderly women. Gender.

\section{Introdução}

A população mundial de idosos cresce em ritmo acelerado a cada ano. No Brasil, segundo dados do IBGE, houve um aumento na média de vida, passando esta de 45,5 anos no ano de 1940 para 73,1 anos em 2010, ocasionado principalmente pela melhora nas condições gerais de vida (Santos, Andrade \& Bueno, 2009).

0 processo de envelhecimento é um acontecimento natural do desenvolvimento do ser humano, acompanhado por mudanças em dimensões biológicas, psicológicas e socioculturais (FernándezBallesteros, 2011; Prieto-Flores, Moreno-Jiménez, Ferandez-Mayoralas, Rojo-Perez \& Forjaz, 2012; Santos el al., 2009). Para Moraes, Moraes e Lima (2010) o envelhecimento é composto por duas dimensões distintas: o envelhecimento somático e o envelhecimento psíquico, em que cada indivíduo apresenta respostas próprias com características específicas, dependendo do grau de fragilidade do organismo e do psiquismo.

Esses graus de fragilidade interferem diretamente na qualidade de vida e no bem-estar deste idoso, já que as dimensões desta qualidade envolvem, além de outros fatores, o status de saúde (atividades de lazer, relacionamento com vizinhos e finanças), satisfação com a saúde (mobilidade, atividades usuais, mobilidade e satisfação com os vizinhos) e satisfação com a vida (idade, família e saúde) (Prieto-Flores et al., 2012). Tais fatores, quando não administrado de forma saudável, poderão influenciar as doenças crônicas, como, por exemplo, a depressão (Carneiro et al., 2007; Leite, Carvalho, Barreto \& Falcão, 2006), cardiopatias, diabetes, artrite, osteoporose (Chittleborough, Winefield, Gill, Koster \& Taylor, 2011), câncer (Hoeymans et al., 1999), causando não apenas uma limitação funcional, mas implicando também no bem-estar dos idosos (Wikman, Wardle, \& Steptoe, 2011) e do seu entorno psicossocial.

Para Chittleborough et al. (2011), existe uma relação entre a presença de doenças crônicas e estresse psicológico, para o qual a idade é um fator importante, justamente porque as pessoas passam por diferentes experiências em cada estágio da vida (diferentes estressores, prioridades, estado de saúde). Especificamente, essa relação existe porque o idoso apresenta perda de resposta emocional que provoca sintomas como diminuição de prazer nas atividades habituais, vontade de ficar isolado em casa, alteração de sono, pensamentos constantes sobre o passado e perda de energia (Gazalle, Lima, Tavares, \& Hallal, 2004). Segundo Duarte e Rego (2007), o idoso que apresente mais de três dessas patologias crônicas poderia se incluir, diagnosticamente, em uma sintomatologia depressiva.

De forma geral, a depressão é uma alteração de comportamento que causa ou é causada por um isolamento social e que com frequência acomete idosos (Pinho, Custódio, \& Makdisse, 2009; Rocha, Alves, Silva \& Vieira, 2005). É uma doença psiquiátrica muito comum entre os idosos e frequentemente não é diagnosticada, portanto, não recebe tratamento adequado. As mudanças psicológicas que ocorrem no decorrer do envelhecimento são: dificuldade à adaptação a novos papéis e mudanças rápidas, planejamento do futuro, falta de motivação, enfrentamento de perdas orgânicas, afetivas e sociais, depressão, suicídio, perda ou diminuição de autoimagem e autoestima (Zimerman, 2000). É então um problema de saúde pública (Melo \& Teixeira, 2011; Silberman et al., 1995), já que a alta prevalência e a associação com doenças crônicas alteram a qualidade de vida (Djernes, 2006; Penninx, Deeg, Eijk, Beekman \& Guralnick, 2000) e aumentam o risco de depressão e, possivelmente, de suicídio (Chang-Quan et al., 2010).

Sendo assim, os fatores de risco associados à depressão podem ser agrupados em fatores sociodemográficos (sexo, idade, situação conjugal, escolaridade, condição socioeconômica e condições de moradia), suporte social, eventos de vida estressores, morbidades psiquiátricas (histórico psiquiátrico prévio e familiar, comorbidades psiquiátricas, personalidade, abuso ou dependência do uso de 
álcool, distúrbios do sono e déficits cognitivos) e condições de saúde, diminuindo, com isso, a qualidade de vida (Pinho et al., 2009).

A avaliação da qualidade de vida (QV) apresenta inúmeros aspectos subjetivos. Por tal condição, faz-se necessário mensurá-la com instrumentos confiáveis; estes, por sua vez, sugerem uma avaliação completa dos indivíduos, realizada desde ensaios clínicos até avaliação dos serviços de saúde (Velarde-Jurado \& Avila-Figueroa, 2002).

Com isso, Verdugo, Schalock, Keith e Stancliffe (2005) discutiram alguns princípios que devem guiar a avaliação de qualidade de vida (processos de medidas e interrogatórios). Segundo esses autores, a qualidade de vida é um constructo multidimensional formada por componentes objetivos e subjetivos. A pessoa que responde deve ser o avaliado, mesmo na presença de desabilidades intelectual ou a própria família do avaliado, utilizar sempre métodos qualitativos ou quantitativos (pluralismo metodológico), ser sensível às diferenças culturais e no fato dos sistemas organizacionais mudarem o objetivo dos indicadores de qualidade de vida.

Segundo Farquhar (1995), é possível encontrar um número considerável de escalas que avaliam a qualidade de vida. Elas variam no conceito, conteúdos e na sua construção psicométrica, dessa forma, não podem ser comparadas de forma direta. Sendo assim, de acordo com o autor supracitado, os idosos são aqueles que estão habilitados a falar sobre qualidade de vida, pois não relatam apenas do lado bom, mas também citam acontecimentos negativos da vida, os quais interferem no desenvolvimento social, afetivo e comportamental da vida dessas pessoas.

Dentre os instrumentos validados para população idosa brasileira, pode-se referenciar o SF-36 e o WOOHOL Old. O primeiro instrumento - SF-36 (Medical Outcomes Study 36-item Health Survey Short-Form) - foi criado a partir do Medical Outcomes Study (MOS) e trata-se de um questionário publicado em inglês em 1990, traduzido e validado em vários idiomas e culturas, com aproximadamente 40 países fazendo uso desse instrumento (Ware \& Gandek, 1998). Ele possui 36 itens organizados em oito escalas e que podem ser agrupados em dois componentes: físico e mental. 0 instrumento permite mensurar dimensões variadas de saúde, permitindo também avaliar o impacto da doença, bem como os benefícios do tratamento na saúde do respondente. No Brasil, o SF-30foi traduzido e validado por Ciconelli, Ferraz, Santos, Meinão e Quaresma (1999) em um estudo envolvendo indivíduos com artrite reumatóide, sendo assim, considerado adequado para a administração nas condições socioeconômicas e culturais da população brasileira;

O segundo instrumento, WOQHOL OLD, é composto por 24 itens distribuídos em seis facetas: funcionamento dos sentidos, autonomia, atividades passadas, presentes e futuras, participação social, morte e morrer, e intimidade. Validado no Brasil por Fleck, Chachamovich e Trentine (2006), o módulo WHOQOL-OLD representa uma alternativa útil e com bom desempenho psicométrico na investigação de qualidade de vida em idosos.

Segundo Hedrick (1995), apesar da existência de instrumentos (testes e escalas) contribuírem para a avaliação da qualidade de vida, estes não devem substituir o exame físico, sendo possível utilizar ambas as formas de avaliação quando se pretende atuar na área da saúde geriátrica,visto que, na geriatria, quando se trata de avaliar a qualidade vida de idosos, deve-se fazê-lo de forma ampla e cuidadosa, avaliando todas as dimensões que são afetadas pelo processo de envelhecimento (como por exemplo, capacidade funcional, aspectos físicos, psicológicos, sociais e cógnitos) (Paixão Júnior \& Reichenheim, 2009). Ainda que exista tal sugestão no uso de instrumento qualitativos e quantitativos para avaliar o desenvolvimento da saúde do idoso, no Brasil existem apenas dois instrumentos validados e capacitados para tal condição: os citados anteriormente, o SF-36 e o WHOOQOL.

Mesmo com a qualidade desses instrumentos, salienta-se a existência de outros fatores que afetam a qualidade de vida de indivíduos idosos, sendo um desses relacionado ao gênero, uma vez que os estudos demonstram que mulheres apresentam uma maior incidência de depressão, e com isso, uma menor qualidade de vida (Sebastião, Christofoletti, Gobbi. Hamanaka \& Gobbi, 2009). Todavia, é importante caracterizar as variáveis que afetam a qualidade de vida de homens e mulheres de vários segmentos sociais. Sebastião et al. (2009) analisou as possíveis relações e diferenças entre qualidade de vida em idosos através do SF-36, observando que os homens percebem esta de forma melhor em comparação com as mulheres nas dimensões física, mental e na qualidade de vida total. 
Na mesma direção de raciocínio, os resultados encontrados por Pereira et al. (2006) em um estudo com idosos de município Teixeiras - RS revelaram uma pequena diferença entre os sexos nos domínios Físico, Psicológico e Ambiental, sendo os escores médios desses domínios significativamente maiores entre os homens, o que significa que apresentam melhor qualidade de vida que as mulheres. No estudo de Thome, Dykes e Hallberg (2004), o resultado se mantém, isto é, o sexo masculino também apresentou escores melhores no domínio Físico.

Com base em fatores demográficos e socioeconômicos, Lima et al. (2009) avaliaram a qualidade de vida relacionada à saúde de idosos do sudeste brasileiro e observaram em seus resultados que em relação ao sexo, as mulheres apresentaram piores escores que os homens em todos os domínios do SF36, exceto no domínio dos Aspectos Físicos.

Em um estudo na Unidade de Saúde do município de Curitiba, foi realizado um levantamento sobre a qualidade de vida de um grupo de idosos utilizando o instrumento WHOQOL-BREF. Os autores verificaram que não existe diferença significativa entre os escores dos domínios em relação ao sexo. O sexo masculino tem o maior escore em todos os quatro domínios, sendo o maior deles no domínio Psicológico e o menor no Físico. Para o sexo feminino, o maior escore médio apresenta-se também no domínio Psicológico e o menor, no domínio do Meio Ambiente (Balduíno \& Jacopetti, 2009). Porém, seria possível garantir especificamente que os resultados a respeito da qualidade de vida são uma condição sine quo non, apenas do gênero? É possível explicar esse construto - qualidade de vida - apenas por ser homem ou mulher? Estaria a solução somente no biológico?

Sabe-se que o perfil psicológico de gênero tem influência direta nos comportamentos e atitudes do indivíduo, portanto, a forma de se pensar e agir torna o sujeito diferenciado dependendo da polarização do esquema de gênero. Segundo Bem (1984), a interação do biológico com a cultura, e não somente o biológico, é capaz de determinar as diferenças entre ser masculino e feminino.

Segundo Giavoni (2000), todos os indivíduos, independentemente do sexo, possuem em menor ou maior intensidade fatores da masculinidade e feminilidade. Esta afirmação diferencia a autora de Bem (1984), uma vez que Bem subdivide os indivíduos em esquemáticos masculinos, esquemáticos femininos, andróginos e aesquemáticos.

Giavoni (2000) afirma que dependendo dessa maior e menor intensidade, os indivíduos podem ser subdivididos da seguinte forma: Heteroesquemáticos Masculino (HM) - em que haverá um predomino do esquema masculino sobre o feminino; os Heteroesquemáticos Femininos - em que haverá um predomino do esquema feminino sobre o masculino; e os Isoesquemáticos, que possuem o equilíbrio entre os esquemas de gênero.

Mesmo não utilizando a proposta teórico-metodológica de Giavoni (2000) sobre o esquema de gênero, e com base na avaliação do perfil psicológico através do inventário de Papéis Sexuais de Bem (BSRI), Moore, Kreitler, Ehrenfeld e Giladi (2005) avaliaram a qualidade de vida de pacientes com Síndrome de Parkinson e observaram que os indivíduos andróginos apresentaram melhor níveis de qualidade de vida em comparação com indivíduos esquemáticos masculinos, esquemáticos femininos e indiferenciados.

Para Pikler e Brown (2010), os perfis de gênero de pacientes com câncer influenciam na sua qualidade de vida, uma vez que os resultados do estudo desses autores revelaram que sujeitos esquemáticos masculinos e aesquemáticos possuem uma probabilidade de desenvolver sintomas de estresse maior do que sujeitos com esquema feminino e andróginos. Essa possibilidade de desenvolvimento de estresse os leva a piores níveis de qualidades de vida, o que corrobora os resultados encontrados por Moore et al. (2005).

$\mathrm{Na}$ literatura brasileira só foi encontrado um estudo que avaliou a qualidade de vida de idosas comparando-as pelos perfis psicológicos de gênero e utilizando para isso o Modelo Interativo de Giavoni (Giavoni, 2000). Trata-se do estudo de Santana (2011), no qual se observou que mulheres idosas heteroesquemáticas masculinas e isoesquemáticas apresentam maiores níveis de qualidade de vida quando comparadas com heteroesquemáticas femininas. É possível que essa diferença com relação aos estudos americanos de Moore et al. (2005) e Pikler e Brown (2010) deva-se às diferenças entre as duas culturas e ao foco teórico abordado pelos autores.

Em recente pesquisa realizada nos sites de busca (Index Psi, 2012; Scielo, 2012), com as palavras-chave "esquema de gênero", "qualidade de vida", "idoso e perfil de gênero", "saúde" e "terceira idade", não 
foi encontrado nenhum estudo que avaliasse as variáveis aqui abordadas, tampouco o modelo teórico proposto entre elas. Desta forma, o presente estudo tem como objetivo verificar, a partir de uma análise de modelagem de equação estrutural, a associação entre as variáveis perfil psicológico de gênero, nível de depressão e a qualidade de vida em mulheres idosas brasileiras.

\section{Método}

Amostra

A amostra foi composta por 143 mulheres idosas participantes de um Centro de Convivência de Idosos do Distrito Federal. Destas, $63 \%$ eram casadas, com idade média de 67,45 $\pm 3,87$ anos e nível de escolaridade igual ou superior ao fundamental completo (35\%), de várias naturalidades, residentes do Distrito Federal.

\section{Instrumentos}

Para a avaliação da QV dos idosos foi utilizado o Medical Outcomes Study 36 (SF-36), que foi validado no Brasil por Ciconelli et al. (1999). É um instrumento genérico de avaliação de QV, tanto da população em geral quanto de idosos, que apresenta fácil administração e compreensão e não é muito extenso. É multidimensional e possui 36 itens subdivididos em oito domínios: Capacidade Funcional (10 itens), que avalia a presença e a extensão de limitação relacionada à capacidade física; Aspectos Físicos (4 itens), que avaliam o impacto negativo da saúde física no desempenho das atividades diárias e/ou profissionais; Aspectos Emocionais (3 itens), que avaliam o reflexo das condições emocionais no desempenho das atividades diárias e/ou profissionais; Dor (2 itens), que avalia a presença de dor, sua intensidade e sua interferência nas atividades da vida diária; Estado Geral de Saúde (5 itens), que avalia a percepção subjetiva do estado geral de saúde; Vitalidade (4 itens), que avalia itens que consideram o nível de energia e de fadiga para realização de tarefas do cotidiano; Aspectos Sociais (2 itens), que analisam a integração do indivíduo em atividades sociais; e Saúde Mental (5 itens), que inclui questões sobre ansiedade, depressão, alterações no comportamento ou descontrole emocional e bem estar psicológico. 0 questionário apresenta ainda uma questão de avaliação comparativa entre as condições de saúde atual e de um ano atrás e avalia tanto aspectos negativos de saúde (doença ou enfermidade), como aspectos positivos (bem-estar). Cada escala recebe um escore que varia de 0 a 100, em que 0 representa o pior resultado e 100 representa o estado perfeito de saúde (Ware, \& Sherboune, 1992).

O Inventário Feminino dos Esquemas de Gêneros do Autoconceito (IFEGA) foi utilizado para classificar as idosas segundo o perfil tipológico. 0 instrumento é composto por itens agrupados em fatores, que compõem o esquema masculino (EM) e o esquema feminino (EF) e foi elaborado e validado por Giavoni e Tamayo (2005). O IFEGA apresenta 75 itens que são avaliados por meio de uma escala de quatro pontos e avalia três aspectos do EM (arrojamento, $\alpha=0$,87; egocentrismo, $\alpha=0,83$; e negligência, $\alpha=0,73$ ) e três aspectos do EF (sensualidade, $\alpha$ $=0,92$; inferioridade, $\alpha=0,82$; e ajustamento social, $\alpha=0,77)$. 0 instrumento é capaz de avaliar o perfil psicológico do indivíduo nas dimensões que compõem tanto o EM quanto o EF, permitindo classificar os indivíduos nos grupos tipológicos de esquemas de gênero em Heteroesquemático Masculino, Heteroesquemático Feminino e Isoesquemático.

A Escala de Depressão para Idosos (EDI) (Giavoni, Melo, Parente \& Dantas (2008). A EDI é composta por dois fatores principais: fator cognitivo-afetivo ( $\mathrm{n}=27$ itens) e fator somático-motor ( $\mathrm{n}=11$ itens) que resulta em um total de 38 itens, avaliados mediante escala do tipo Likert de cinco pontos, em que o escore 1 indica que o item não se aplica e o escore 5 indica que o item se aplica totalmente ao respondente. A escala proporciona, ainda, um fator de segunda ordem, denominado nível geral de depressão ( $\mathrm{n}=38$ itens) que reúne em si os itens dos dois fatores principais. Os escores de cada fator devem ser obtidos pela média aritmética.

\section{Procedimentos}

As voluntárias foram reunidas em um auditório e a estas foi explicado o objetivo da pesquisa, situação em que aquelas que concordaram em participar da amostra assinaram o Termo de Consentimento Livre e Esclarecido. Após a assinatura, foram agendados 
horários para cada idosa, as quais compareceram ao local da pesquisa para aplicação da EQV, SF-36, IFEGA e EDI, bem como para o preenchimento do cadastro dos dados socioeconômicos. Este procedimento teve duração variável de 10 minutos a 30 minutos por pessoa a cada encontro agendado.

Todos os procedimentos adotados nesta pesquisa seguiram as orientações previstas na Resolução 196/96 do CNS e na Resolução 016/2000 do Conselho Federal de Psicologia (CNS, 1996; ANPEPP, 2000). Em todas as análises foi estipulado uma margem de erro de $5 \%$.

Este projeto foi aprovado pelo Comitê de Ética da Universidade Católica de Brasília (050/2010).

\section{Análise de dados}

No que se refere à análise dos dados desta pesquisa, utilizou-se a versão 17.0 do pacote estatístico SPSS para Windows. Foram computadas estatísticas descritivas (tendência central e dispersão); no AMOS 16.0 foram computados e avaliados os indicadores estatísticos para o Modelo de Equações Estruturais (MEE) considerado, segundo uma bondade de ajuste subjetiva, os seguintes indicadores: $\mathrm{c}^{2}$ /gl (grau de liberdade), que admite como adequados índices entre 2 e 3, aceitando-se até 5; Raiz Quadrada Média Residual (RMR), que indica o ajustamento do modelo teórico aos dados, na medida em que a diferença entre os dois se aproxima de zero; índices de qualidade de ajuste, dados pelos GFI/AGFI, que medem a variabilidade explicada pelo modelo, e com índices aceitáveis a partir de 0,80 ; CFI, que compara de forma geral o modelo estimado e o modelo nulo, considerando valores mais próximos de 1 como indicadores de ajustamento satisfatório e o RMSEA, que refere-se ao erro médio aproximado da raiz quadrática, que deve apresentar intervalo de confiança como ideal situado entre 0,05 e 0,08 (Byrne, 2001; Hair, Tatham, Anderson \& Black, 2005; Joreskög \& Sörbom, 1989).

As técnicas da análise da modelagem estrutural têm a clara vantagem de levar em conta a teoria para definir a associação entre as variáveis hipotetizadas, bem como apresentar indicadores de bondade de ajuste que permitem decidir objetivamente sobre a validade de construto da medida analisada. Dois resultados principais podem ser esperados ao trabalhar com a análise estrutural:
- Estimativa da magnitude dos efeitos estabelecida entre variáveis, as quais estão condicionadas ao fato de o modelo especificado (isto é, o diagrama) estar correto;

- Teste que verifica se o modelo é consistente com os dados observados a partir dos indicadores estatísticos, podendo dizer que resultado, modelo e dados são plausíveis, embora não se possa afirmar que este é correto (Farias \& Santos, 2000). Atende-se, assim, não a certeza total do modelo, mas a sua probabilidade sistemática na relação entre as variáveis.

Um dos principais objetivos das técnicas multivariadas - neste caso, considera-se a modelagem de equação estrutural - é expandir a habilidade exploratória do pesquisador e a eficiência estatística e teórica no momento em que se quer provar a hipótese levantada no estudo. Apesar das técnicas estatísticas tradicionais compartilharem de limitações, nas quais é possível examinar somente uma relação entre as variáveis, é de suma importância para o pesquisador o fato de ter relações simultâneas, afinal, em alguns modelos existem variáveis que são independentes em algumas relações e, dependentes em outras.

De acordo com Farias e Santos (2000), Hair et al. (2005) e Zamora e Lemus (2008), ao considerar a modelagem estrutural do modelo - isto é, a análise de caminhos (path analysis) - relaciona-se as medidas de cada variável conceitual como confiáveis, acreditando que não existe erro de medida (mensuração) ou de especificação (operacionalização) das variáveis; cada medida é vista como exata manifestação da variável teórica. Assim considerado, desenha-se o modelo teórico que se pretende tomar a partir elaboração hipotética entre as variáveis independente e dependente, isto é, entre as variáveis latentes e variáveis observáveis, por exemplo: no desenvolvimento desse modelo - a elaboração da ligação entre as figuras que caracterizam as variáveis estudadas - um retângulo é considerado como variável observada medida pelo pesquisador; uma elipse é considerada variável latente, isto é, construto hipotético não observado; uma seta com uma ponta indica o caminho ou a relação causal entre duas variáveis; uma seta com duas pontas representa a covariância, isto é, que estas variáveis se associam entre si; por fim, uma pequeno círculo preenchido por um número e letra 
refere-se a um erro de medida. A partir do momento em que se elabora a hipótese, identifica-se cada uma dessas figuras associando as variáveis que se quer provar a múltipla influência.

\section{Resultados}

A fim de atender ao objetivo principal do presente estudo, o qual tratou de verificar um modelo teórico entre perfil psicológico de gênero, qualidade de vida e depressão em idosas, considerando um modelo recursivo de equações estruturais, gerou-se no AMOS GRAFICS 16.0 um modelo mais geral (tomando cada construto com seus respectivos fatores - ver seção método). Este apresentou pesos (saturações) que explicam o modelo na seguinte direção: o perfil de gênero associou-se positivamente a depressão e negativamente a qualidade de vida. Neste modelo observaram-se indicadores psicométricos que estavam de acordo com a exigência na literatura estatística sobre modelagem estrutural, mas o modelo em si tangencia ao que teórica, metodológica e estatisticamente se esperava da associação entre os fatores e seu respectivo construto, isto é, ao gerar os modelos, não se aceita saturação negativa entre eles as variáveis (componentes) dos construtos (Hoe, 2008). Esse fato não inviabiliza o modelo pretendido, mas orientou uma nova direção hipotética, ainda convergindo para o modelo que se pretendia, buscando com isso ser mais parcimonioso.

Sendo assim, a partir desses resultados, resolveu-se excluir os fatores dos construtos que se associaram negativamente entre eles (neste caso, do construto qualidade de vida, excluiu-se o fator [componente], dor, estado geral de saúde, vitalidade e saúde mental) e gerou-se um novo modelo salientado a perspectiva teórica, com base em um maior esclarecimento da definição das variáveis. Desta forma, seguindo a direção teórica e estatística do cálculo de modelagem estrutural, procurou-se com parcimônia gerar um modelo que não considerasse algumas variáveis quanto construto e sim como variáveis observáveis, especialmente as variáveis relacionadas ao perfil de gênero (por exemplo, considerou-se, arrojamento, egocentrismo e negligência [perfil masculino] e sensualidade, inferioridade e ajustamento social [perfil feminino]), de acordo com o destacado na sessão análise de dados do presente estudo.

Tabela 1- Indicadores Psicométricos dos Modelos Teóricos

\begin{tabular}{|c|c|c|c|c|c|c|}
\hline Modelos & $\chi^{2} / g 1$ & GFI & AGFI & CFI & TLI & $\begin{array}{c}\text { RMSEA } \\
\text { (intervalo) }\end{array}$ \\
\hline $\begin{array}{l}\text { Modelo norma perfil } \\
\text { Masculino" }\end{array}$ & 1,01 & 0,98 & 0,95 & 0,99 & 0,99 & $\begin{array}{c}0,02 \\
(0,00-0,10)\end{array}$ \\
\hline Modelo causal 1* & 1,27 & 0,97 & 0,93 & 0,99 & 0,98 & $\begin{array}{c}0,04 \\
(0,00-0,10)\end{array}$ \\
\hline Modelo causal 2** & 1,04 & 0,98 & 0,95 & 0,99 & 0,99 & $\begin{array}{c}0,02 \\
(0,00-0,09)\end{array}$ \\
\hline Modelo causal $3^{* * *}$ & 1,15 & 0,97 & 0,94 & 0,99 & 0,98 & $\begin{array}{c}0,03 \\
(0,00-0,10)\end{array}$ \\
\hline $\begin{array}{l}\text { Modelo norma perfil } \\
\text { feminino }^{\# \#}\end{array}$ & 1,21 & 0,98 & 0,94 & 0,99 & 0,98 & $\begin{array}{c}0,04 \\
(0,00-0,11)\end{array}$ \\
\hline Modelo causal $4^{* * * *}$ & 1,23 & 0,98 & 0,94 & 0,99 & 0,98 & $\begin{array}{c}0,04 \\
(0,00-0,12)\end{array}$ \\
\hline Modelo causal $5^{* * * * *}$ & 1,73 & 0,96 & 0,91 & 0,97 & 0,94 & $\begin{array}{c}0,06 \\
(0,00-0,12)\end{array}$ \\
\hline Modelo causal $6^{* * * * * *}$ & 1,22 & 0,98 & 0,94 & 0,99 & 0,98 & $\begin{array}{c}0,04 \\
(0,00-0,12)\end{array}$ \\
\hline
\end{tabular}

Notas: "Somatório dos fatores do perfil de gênero masculino; Perfil de gênero masculino de arrojamento, qualidade de vida e depressão; **Perfil de gênero masculino egocêntrico, qualidade de vida e depressão; ***Perfil de gênero masculino negligente, qualidade de vida e depressão; \#\#Somatório dos fatores do perfil de gênero feminino; ****Perfil de gênero feminino ajustamento, qualidade de vida e depressão; ${ }^{* * * * *}$ Perfil de gênero feminino inferioridade, qualidade de vida e depressão; ****Perfil de gênero feminino sensualidade, qualidade de vida e depressão. $p$ > 0,05. 
Feita essa escolha, procurou-se então gerar os modelos específicos. Para isso, considerou-se isoladamente cada variável do perfil de gênero de masculinidade e feminilidade associados às variáveis qualidade de vida e depresão. Na Tabela 1, esses resultados podem ser observados para os respectivos modelos.

De acordo com a tabela acima, os indicadores psicométricos estão dentro do intervalo exigido pela literatura vigente. Após as modificações dos erros de ajustes e tendo todas as saturações (lambdas $\lambda$ ) estatisticamente diferentes de zero ( $t>1,96, \mathrm{p}<$ $0,05)$, observou-se os seguintes resultados para os respectivos modelos:

No que se refere ao modelo geral do perfil de gênero quanto à norma masculina (somatório dos fatores de perfil de gênero masculino), observou-se que esta variável influenciou, com lambdas fracos, a qualidade de vida $(\lambda=0,06)$ e a depressão $(\lambda$ $=0,02)$, mas essas duas últimas variáveis se associaram negativamente, $(\lambda=-0,18)$; de forma específica, no modelo 1 , o perfil de gênero masculino de arrojamento associou-se positivamente $(\lambda=$ $0,15)$ à qualidade de vida e a depressão $(\lambda=0,03)$, porém, apresentou uma associação fraca; todavia, deve-se destacar a existência de uma associação negativa entre qualidade de vida e a depressão $(\lambda=$ -0,18); em relação ao modelo 2 , o perfil de gênero masculino de egocentrismo associou-se, negativamente $(\lambda=-0,17)$ à qualidade de vida e a depressão $(\lambda=0,03)$, também com lambda associativo fraco, destacando uma associação negativa da qualidade de vida e com a depressão $(\lambda=-0,16)$; quanto ao modelo 3 , o perfil de gênero masculino de negligente associou-se de forma fraca e negativamente $(\lambda=-0,04)$ à qualidade de vida e, também, com um lambda fraco com a depressão $(\lambda=0,03)$, contudo, houve uma associação negativa da qualidade de vida e com a depressão $(\lambda=-0,14)$;

No que diz respeito ao modelo geral do perfil de gênero quanto à norma feminina (somatório dos fatores de perfil de gênero feminino), ela influencia positivamente a qualidade de vida $(\lambda=0,17) \mathrm{e}$ negativamente a depressão $(\lambda=-0,05)$; especificamente, no modelo 4 , o perfil de gênero feminino de ajustamento associou-se positivamente, mas com um lambda fraco $(\lambda=0,05)$ para com a qualidade de vida e para a depressão $(\lambda=0,08)$. No entanto, para essas duas ultimas variáveis - a qualidade de vida e a depressão - observou-se uma associação negativa $(\lambda=-0,17)$; quanto ao modelo 5 , o perfil de gênero feminino de sensualidade associou-se positivamente $(\lambda=0,10)$ à qualidade de vida e à depressão $(\lambda=$ $0,05)$, porém, com uma associação fraca, mas houve uma associação, negativa da qualidade de vida e com a depressão $(\lambda=-0,12)$. Por fim, o modelo 6 , referente ao perfil de gênero feminino de inferioridade, associou-se negativamente $(\lambda=-0,30)$ à qualidade de vida e positivamente à depressão $(\lambda=0,20)$, tendo essas duas últimas variáveis se associado negativamente entre si $(\lambda=-0,14)$.

\section{Discussão}

De forma geral, os modelos propostos revelam que, em todos eles, independente do perfil de gênero, a qualidade de vida foi capaz de inibir a depressão, especialmente quando o construto da qualidade de vida contempla dimensões como capacidade funcional, aspectos físicos, aspectos emocionais e aspectos sociais.

Considerando a norma masculina, destaca-se que esta apresentou menor poder explicativo para a qualidade de vida e a depressão do que a norma feminina, pois esta última, não somente apresentou uma melhor associação com qualidade de vida, mas também, apesar de um lambda fraco, associou-se negativamente à depressão. Todavia, faz-se necessário salientar cada modelo verificado, pois para cada um deles, com a especificidade dos fatores do perfil gênero masculino ou feminino estabelecido como variável observada, requer uma interpretação mais apurada.

Ao considerar a influência do arrojamento na qualidade de vida e depressão, observou-se que esta dimensão do perfil masculino tem uma força explicativa apenas em uma direção (a qualidade de vida), não sendo tão forte para a depressão. Um raciocínio semelhante pode ser feito para a dimensão de egocentrismo, pois ele não tem poder explicativo para depressão, mas, por outro lado, associa-se negativamente à qualidade de vida. Isto é, se por um lado, o arrojamento contribui para que os sujeitos melhorem a sua vida, por outro, o egocentrismo, faz o caminho inverso. Por fim, em relação à dimensão negligente, esta não apresentou lambdas associativos fortes, tanto para qualidade de vida quanto para a depressão, permitindo pensar se essa dimensão contribuiria para ambas as variáveis dependentes do modelo. 
No que diz respeito às dimensões do perfil de gênero feminino, o ajustamento e a sensualidade se associaram de forma fraca, mesmo que com uma associação positiva, mas a dimensão inferioridade foi a que apresentou melhores associações, revelando o quanto a aderência a ela poderá inibir a qualidade de vida (justamente por ter se associado negativamente) e contribuir para depressão (pois associou-se positivamente).

De forma geral, prestar atenção ao perfil de gênero, principalmente à aderência das idosas a determinada dimensão da masculinidade ou feminilidade não somente contribuiria para compreender o como, o porquê e onde, como também seria mais fácil de trabalhar atividades de ocupação ou atividade física e de lazer que melhorassem a qualidade de viver das idosas do estudo - bem como, de outras idosas - e inibir a depressão.

Algo merece ser destacado, principalmente quanto à exclusão dos fatores da qualidade de vida nessa amostra, pois se trata de uma amostra homogênea, de idosas participantes de uma instituição de ensino superior privado de Brasília - DF há anos e realizam atividades físicas, recreacionais e educativas, apresentando-se estáveis fisicamente e psicologicamente. Assim, seria de grande utilidade desenvolver um estudo que enfatizasse diferentes amostras (por exemplo, adolescentes, jovens adultos e idosos que façam parte de programas de intervenção e os que não participem desses programas), a fim de comparar não somente a consistência do modelo, mas também a evolução das pessoas nas variáveis abordadas neste estudo, especialmente na depressão e qualidade; outro estudo que mereceria atenção seria o da avaliação das mesmas variáveis deste estudo com sujeitos clínicos inclusos ou não em programas de intervenção semelhantes.

\section{Referências bibliográficas}

Balduíno, E., \& Jacopetti, S. R. (2009). Levantamento da qualidade de vida de um grupo de idosos. Boletim de Enfermagem, 3(2), 31-47.

Bem, S. L. (1984). Androgyny and gender schema theory: a conceptual and empirical integration. In R. A. Dienstbier (ed.). Nebraska Symposium on Motivation (Vol 32, pp. 179-226). Nebraska: Nebraska University Press.
Bem, S. L. (1974).The measurement of psychological androgyny. Journal of Consulting and Clinical Psychology, 429(2), 155-62.

Byrne, B. M. (2001).Structural Equation Modeling With AMOS, EQS, and LISREL: Comparative Approaches to Testing for the Factorial Validity of a Measuring Instrument. International Journal of Testing, 1(1), 55-86.

Carneiro, R. S., Falcone, E., Clark, C., Prette, Z. D., \& Prette, A. D. (2007). Qualidade de Vida, Apoio Social e Depressão em Idosos: Relação com Habilidades Sociais. Psicologia: Reflexão e Crítica, 20(2), 229-237.

Chang-Quan, H. et al. (2010). Health status and risk for depression among the elderly: a meta-analysis of published literature. Age and Ageing, 39(1), 23-30.

Chittleborough, C. R., Winefield, H., Gill, T. K., Koster, C., \& Taylor, A. W. (2011). Age differences in associations between psychological distress and chronic conditions. International Journal of Public Health, 56(1), 71-80.

Ciconelli, R. M., Ferraz, M. B., Santos, W., Meinão, I., \& Quaresma, M. R. (1999). Tradução para a língua portuguesa e validação do questionário genérico de avaliação de qualidade de vida SF-36 (Brasil SF-36). Revista Brasileira de Reumatologia, 39(3), 143-150.

Djernes, J. K. (2006). Prevalence and predictors of depression in population of elderly: a review. Acta of Psychiatryc Scandinav, 113(5), 372-87.

Duarte, M. B., \& Rego, M. A. B. (2007). Comorbidade entre depressão e doenças clínicas em um ambulatório de geriatria. Caderno de Saúde Pública, 23(3), 691-700.

Farquhar, M. (1995). Eldery people's definitions of quality of life. Social Science \& Medicine, 41(10), 1439-1446.

Farias, S. A., \& Santos, R. C. (2000). Modelagem de equações estruturais e satisfação do consumidor: uma investigação teórica e prática. Revista de Administração Contemporânea, 4(3), 107-132.

Fernández-Ballesteros, R. (2011). Quality of Life in Old Age: Problematic Issues. Applied Research Quality Life, 6(1) 21-40. 
Fleck, M. P., Chachamovich, E., \& Trentine, C. (2006). Development and validation of the Portuguese version of the WHOQOL-OLD module. Revista de Saúde Pública, 40(5), 785-791.

Gazalle, F. K., Lima, M. S., Tavares, B. F., \& Hallal, P. C. (2004). Sintomas depressivos e fatores associados em uma população idosa no sul do Brasil. Revista de Saúde Pública, 38(3), 365-71.

Giavoni, A., Melo, G. F., Parente, I., \& Dantas, G. (2008). Elaboração e validação da Escala de Depressão para Idosos. Caderno de Saúde Pública, 24(5), 975-982.

Giavoni, A. \& Tamayo, A. (2005). Inventário feminino dos esquemas de gênero do autoconceito (IFEGA). Estudos de Psicologia, 10(1), 25-34.

Giavoni, A., \& Tamayo (2010). The psychological synthesis evaluated by the interactive model. Psicologia: Reflexão e Crítica, 23(3), 593-601.

Hair, J. F., Tatham, R. L., Anderson, R. E., \& Black, W. (2005). Análise Multivariada de Dados. Porto Alegre: Bookman.

Hedrick, S. C. (1995). Assessment of functional status: activities of daily living. In Rubenstein, L. Z., Wieland, D., Bernabei, R. (ed.). Geriatric assessment technology: state of the art. Milano: Editrice Kurtis.

Hoe, S. L. (2008). Issues and procedures in adopting structural equation modeling technique. Journal of Applied Quantitative Methods, 3(1): 76-83.

Hoeymans et al. (1999). The Contribution of Chronic Conditions and Biol Disabilities to Poor Self-Rated Health in Elderly Men. Journal of Gerontology Series A: Biological Sciences and Medical Sciences, 54(10), M501-M506.

Joreskög, K., \& Sörbom, D. (1989). LISREL 7 user's reference guide. Mooresville: Scientific Software.

Leite, V. M. M., Carvalho, E. D. F., Barreto, K. M. L., \& Falcão, I. V. (2006). Depressão e envelhecimento: estudo nos participantes do Programa Universidade Aberta à Terceira Idade. Revista Brasileira de Saúde Materno Infantil, 6(1), 31-38.
Lima, M. G., Barros, M. B. A., César, C. L. G, Goldbaum, M., Carandina, L., \& Ciconelli, R. M. (2009). Qualidade de vida relacionada à saúde em idosos, avaliada com o uso do SF-36 em estudo de base populacional. Caderno de Saúde Pública, 25(10), 2159-2167.

Melo, E., \& Teixeira, M. B. (2011). Depressão em idosos. Revista SAÚDE, 5(1), 42-53.

Moore, O., Kreitler, S., Ehrenfeld, M., Giladi N. (2005). Quality of life and gender identity in Parkinson's disease. Journal of Neural Transmission, 112(11), 1511-1522.

Moraes, E. M., Moraes, F. L., \& Lima, S. P. P. (2010). Características biológicas e psicológicas do envelhecimento. Revista de Medicina de Minas Gerais, 20(1), 67-73.

Paixão Júnior, C. M., \& Reichenheim, M. E. (2009). Uma revisão sobre instrumentos de avaliação do estado funcional do idoso. Caderno de Saúde Pública, 21(1), 7-19.

Penninx, B., Deeg, D. S. H., Eijk, J. T. M. V., Beekman, A. T. F., \& Guralnick, J. M. (2000). Changes in depression and physical decline in older adults: a longitudinal perspective. Journal of Affect Disorders, 61(1-2), $1-12$.

Pereira, R. J. et al. (2006).Contribuição dos domínios físico, social, psicológico e ambiental para a qualidade de vida global de idosos. Revista de Psiquiatria do Rio Grande do Sul, 28(1), 27-38.

Pikler,V. I., Brown, C. (2010). Cancer Patients' and Partners' Psychological Distress and Quality of Life: Influence of Gender Role. Journal of Psychosocial Oncology, 28(1), 43-60.

Pinho, M. X., Custódio, O., Makdisse, M. (2009). Incidência de depressão e fatores associados em idosos residentes na comunidade: revisão de literatura. Revista Brasileira de Geriatria e Gerontologia, 12(1), 123-140.

Prieto-Flores, M. E., Moreno-Jiménez, A., FernandezMayoralas, G., Rojo-Perez, F., \& Forjaz, M. J. (2012). The Relative Contribution of Health Status and Quality of Life Domains in Subjective Health in Old Age. Social Indicators Research, 106(1), 27-39. 
Rocha, K. M. F., Alves, J. W., Silva, A. S., \& Vieira, D. T. C. (2005). Depressão no idoso e alterações posturais: contribuições fisioterápicas. Revista Multidisciplinar, 2, 22-28.

Santana, M. C. (2011). Perfil tipológico de gênero e a sua relação com risco de quedas, qualidade de vida e percepção do envelhecimento. Dissertação de Mestrado, Universidade Católica de Brasília, Brasília - DF.

Santos, F. H., Andrade, V. M., Bueno, O. F. A. (2009). Envelhecimento: um processo multifatorial. Psicologia em Estudo, 14(1), 3-10.

Sebastião, E., Christofoletti, G., Gobbi, S., Hamanaka, A.Y.Y., \& Gobbi, L.T.B.G. (2009). Atividade física, qualidade de vida e medicamento em idosos: diferenças entre idade e gênero. Revista Brasileira de Cineantropometria e Desempenho Humano, 11(2), 210-216.

Silberman, C., et al. (1995). Cognitive Deficit and depressive symptoms in a community group of elderly people: a preliminary study. Revista de Saúde Pública, 29(6), 444-50.

Thome, B., Dykes, A. K., \& Hallberg, I. R. (2004). Quality of life in old people with and without cancer. Qualife of Life Research, 13(6),1067-80.
Velarde-Jurado, E., \& Avila-Figueroa, C. (2002). Evaluación de la calidad de vida. Salud Pública de México, 44(4), 349-361.

Verdugo, M. A., Schalock, R. L., Keith, K. D., \& Stancliffe, R. J. (2005). Quality of life and its measurements: important principles and guidelines. Journal of Intellectual Disability Research, 49(10), 707-717.

Ware, J. E., \& Gandek, B. (1998). Overview of the SF-36 Health Survey and International Quality of Life Assessment (IQOLA) project. Journal of Clinical Epidemiology, 51, 903-912.

Ware, J. E., \& Sherbourne, C. D. (1992). The MOS 36-Item Short-Form Healthy Survey (SF-36). Medical Care, $30(6), 473-483$.

Wikman, A., Wardle, J., \& Steptoe, A. (2011). Quality of life and affective well-being in middle-aged and older people with chronic medical illnesses: a crosssectional population based study. PLoS One, 6(4), e18952.

Zamora, C. S., \& Lemus, I. S. (2008). Modelos de Ecuaciones Estructurales: Qué es eso? Ciência \& Trabajo, 10(29), 106-110.

Zimerman, G. I. (2000). Velhice - Aspectos biopsicossociais. Porto Alegre: Artmed. 